\title{
Ciência da informação e cognição humana: uma abordagem do processamento da informação
}

\author{
Dulce Amélia Neves \\ Doutora em ciência da informação/UFMG. Professora do Departamento \\ de Biblioteconomia e Documentação da UFPB. \\ E-mail: damelia@jpa.neoline.com.br
}

\section{Resumo}

Apresenta as possibilidades de inter-relação entre a ciência da informação e os estudos relativos à cognição humana. Discute resultados de pesquisas sobre processamento da informação na psicologia cognitiva referentes ao desempenho dos indivíduos na leitura e na aquisição de conhecimento, assim como na transformação de informação em conhecimento. Focaliza estudos sobre a cognição e metacognição e a importância dessa abordagem para a recuperação de informação, assim como o processo de indexação em sistemas de informação.

\section{Palavras-chave}

Ciência da informação. Cognição. Recuperação da informação. Indexação

\section{Information science and human cognition: an information processing approach}

\section{Abstract \\ This article presents the possibilities of relationship between information science and human cognition. In this regard, discusses research results on information processing within the cognitive psychology field. Such studies are concerned with the performance of individuals in reading skills, knowledge acquisition and transforming information onto knowledge. It focus on studies about human cognition and metacognition and the importance of this approach to information retrieval and indexing procedures in the context of information systems.}

\section{Keyword}

Information science. Cognition. Information retrieval. Indexing.

\section{INTRODUÇÃO}

Os últimos anos da segunda metade do século XX foram marcados pela ocorrência de inúmeros estudos nos campos da ciência e da tecnologia que tornaram possível a incorporação de mudanças significativas na produção do conhecimento. Pensada na origem como uma ciência interdisciplinar, a ciência da informação possui, desde seu nascimento, uma ligação íntima com a tecnologia da informação (NEVES; MOURA, 2002). Sua principal função é produzir conhecimentos que contribuam para a solução de problemas relacionados à organização de sistemas de informação especializados na incorporação, sistematização, disseminação e recuperação da informação.

Neste artigo, pretendemos apresentar alguns pontos de interseção entre os estudos da psicologia cognitiva, abordando o processamento da informação e a ciência da informação, visando a efetivar diálogo interdisciplinar.

\section{A CIÊNCIA DA INFORMAÇÃO}

A ciência da informação, assim como os demais campos do conhecimento, precisa incorporar aos processos de formação novas abordagens que permitam o aprimoramento do diálogo entre os sujeitos que interagem com os sistemas de informação, principalmente no que diz respeito aos profissionais da informação e os usuários. Seu objetivo principal é incentivar estudos que promovam conexões entre os sistemas de informação e sujeitos na busca da informação. Para efetuar essa função, a ciência da informação dialoga necessariamente com as áreas do conhecimento que visam a estudos relativos à comunicação humana de modo geral, especificamente organização, representação e uso da informação.

Nos anos 60, a ciência da informação se preocupava com as propriedades e comportamento da informação, com as forças que governam seu fluxo e com os meios de processá-la para facilitar seu acesso e uso. Tentava-se formalizar 


\section{Dulce Amélia Neves}

... as propriedades da informação pela aplicação da teoria da informação, da teoria das decisões e outros construtos da ciência cognitiva, da lógica e/ou da filosofia (SARACEVIC, 1996, p. 16).

Na década de 70, "com o deslocamento do paradigma da recuperação da informação em direção ao usuário e suas interações" (LIMA, 2003, p. 78), a ciência da informação buscou estabelecer um enfoque científico homogêneo, que possibilitasse o estudo dos processos de comunicação nos sistemas de informação. A partir dos anos 80 , a administração foi incluída como parte fundamental da ciência da informação. Já nos anos 90 , volta-se para a prática do profissional da informação, particularmente com estudos voltados para o uso/necessidades da informação e tecnologias da informação.

Como a ciência da informação é um conhecimento que opera com a linguagem, necessário se faz compreender esse fenômeno em sua dinâmica, nos diversos níveis complexos advindos da multiplicidade de perspectivas postas na compreensão do fenômeno informacional. Saracevic (1996) afirma que a ciência da informação é um campo dedicado às questões científicas e às práticas profissionais voltadas para os problemas da efetiva comunicação do conhecimento e de seus registros entre os seres humanos, no contexto social, institucional ou individual, do uso e das necessidades de informação. Entre suas várias funções, está a de descrever intelectualmente a informação. Para Capurro (1991, 3-4),

os seres humanos são processadores biológicos de informação. A informação é realidade duplamente codificada... A ciência da informação pretende estudar a informação em si mesma, ou seja, contribuir para sua análise e sua construção.

A ciência da informação também se propõe a estabelecer uma abordagem científica consistente para o estudo dos vários fenômenos que cercam a noção de informação, sejam eles encontrados nos processos biológicos, na existência humana, ou nas máquinas. Essa busca compreende basicamente a instituição de conjuntos de princípios que orientem o processo de comunicação interna e externa aos sistemas de recuperação da informação, levando em consideração a diversidade comportamental apresentada pelos sujeitos na busca da informação.

Para desempenhar essa função, a ciência da informação dialoga com as áreas que envolvem a efetividade da comunicação humana, o conhecimento da informação e seus registros, as necessidades e os usos da informação, seus contextos sociais, institucionais e individuais. Esse diálogo é, contudo, entrecortado pela complexidade advinda da multiplicidade de perspectivas postas na compreensão do fenômeno informacional (NEVES, 2004; MOURA, 2002).

\section{A COMPREENSÃO DA MENTE}

Nas últimas décadas, ocorreu um incremento nos estudos sobre a cognição humana, principalmente após o surgimento do computador e da modelagem computacional. A ciência cognitiva é uma área de estudos interdisciplinares que se inter-relaciona com psicologia cognitiva, ciência da computação, sistemas de informação, inteligência artificial, neurociências e lingüística, entre outras (LIMA, 2003). A partir dessa inter-relação, as pesquisas desenvolvidas sobre a cognição humana têm buscado apreender o modo como as pessoas pensam, interpretam e percebem o mundo. Estudos sobre a natureza e o desenvolvimento cognitivo dos seres humanos estão voltados para quatro teorias principais do desenvolvimento cognitivo: a de Piaget, a neopiagetiana, a de Vygotsky e a abordagem do processamento da informação.

Para Piaget (1983), a cognição humana é uma forma de adaptação biológica na qual o conhecimento é construído aos poucos a partir do desenvolvimento das estruturas cognitivas que se organizam de acordo com os estágios de desenvolvimento da inteligência. Assim, desenvolvimento cognitivo está ligado aos processos de assimilação e acomodação que promovem o equilíbrio que varia de acordo com a idade (FLAVELL; MILLER, P.H.; MILLER, S.A., 1999; STERNBERG, 2000). Os teóricos neopiagetianos, tomando por base as teorias de Piaget, dão ênfase às habilidades cognitivas, como o processar e coordenar elementos que possibilitam a diferenciação de informações na determinação de subobjetivos para atingir uma meta. Além disso, incluem o conceito de mediação e interação na solução de problemas.

Para Vygotsky (1998), o conhecimento é construído durante as interações entre os indivíduos em sociedade, desencadeando o aprendizado. Assim, processo de mediação se estabelece quando duas ou mais pessoas cooperam em uma atividade, possibilitando uma reelaboração. Nesse sentido, os adultos, que proporcionam modelos de comportamento, organizam e estruturam a participação das crianças em atividades, são denominados "incentivadores cognitivos" na "participação orientada" (FLAVELL, 1979; MILLER, P.H; MILLER, S.A. (1999). 
Entretanto, as crianças não são passivas; assimilam o conhecimento e o reelaboram viabilizando novas atitudes gerando novos comportamentos, sucessivamente.

A teoria do processamento da informação (HUNT, 1980; NEWELL; SIMON, 1972; STERNBERG, 2000) deu origem a grande número de estudos investigando detalhadamente os processos cognitivos envolvidos na resolução de problemas. Tal abordagem vem gradualmente se integrando aos estudos da neurologia, dando origem à neurociência cognitiva. Os teóricos do processamento da informação, de origem mais recente, reúnem diversas abordagens que estudam a mente e a inteligência em termos de representações mentais e seus processos subjacentes ao comportamento observável. Estes pesquisadores consideram o conhecimento como sistema de tratamento da informação. Como afirma Sternberg (2000), os psicólogos do processamento da informação estudam as capacidades intelectuais humanas, analisando a maneira como as pessoas solucionam as difíceis tarefas mentais para construir modelos artificiais que têm por objetivo compreender os processos, estratégias e representações mentais utilizadas pelas pessoas no desempenho destas tarefas.

A abordagem do processamento da informação pesquisa a compreensão textual, conceituada como uma atividade cognitiva que envolve percepção, memória, inferência e dedução. A compreensão do texto ocorre a partir do conhecimento de mundo e da familiaridade com os diversos tipos de textos, requerendo consciência semântica e pragmática de leitores mais proficientes.

Os psicólogos voltados ao estudo do processamento da informação delineiam o processamento do texto na memória do seguinte modo: no primeiro momento, ocorre a percepção da apresentação gráfica, seu arranjo em maiúsculas e minúsculas e as formas cursivas. A seguir, ocorre a tradução das letras em sons, o encadeamento desses sons em uma palavra. Em seguida, o leitor calcula o significado da palavra, repetindo o processo palavra por palavra, até o final do texto. Para tanto, é necessário que o leitor domine os processos léxicos que são usados para identificar as letras e as palavras e para ativar a informação relevante na memória sobre essas palavras (STERNBERG, 2000).

○ processo de compreensão envolve codificação semântica, aquisição de vocabulário, criação de modelos mentais e compreensão das idéias do texto. A codificação semântica é o processo pelo qual a informação sensorial é traduzida em palavras. A aquisição de vocabulário acrescenta ao nosso vocabulário-base novos termos significativos, a partir do contexto ou da consulta a dicionários e enciclopédias, entre outros (STERNBERG, 2000; KOCH; TRAVAGLIA, 2001). Os modelos mentais são um conjunto de proposições que podem levar a mais de um modelo mental e simulam o mundo que nos rodeia e o estado de coisas que faz parte da realidade (JOHNSON-LAIRD, 1983). No caso da leitura, a capacidade de compreensão textual está diretamente relacionada à capacidade de o leitor criar modelos mentais a partir do significado declarado e não-declarado pelo autor do texto. Desse modo, a construção de modelos mentais favorece a compreensão das palavras que lemos e suas combinações, possibilitando o entendimento do significado de um texto em um dado contexto. Segundo Eysenck e Keane (1994) e Seternberg (2000), entre outros, os modelos mentais mais utilizados são os seguintes:

- os esquemas - estruturas cognitivas relacionadas a um conjunto de conhecimentos armazenados em seqüência temporal ou causal, em que são mantidos os conjuntos de características dos objetos e seres que nos rodeiam. Por exemplo: procedimentos para fazer funcionar um aparelho;

- os planos - conjunto de conhecimentos sobre o modo de agir para atingir determinados objetivos. Por exemplo: como fazer para vencer uma partida de xadrez;

- os roteiros ou scripts - ações estereotipadas e predeterminadas aplicadas a situações definidas. Por exemplo, o roteiro aplicado quando vamos ao cinema ou a um restaurante;

- as superestruturas ou esquemas textuais - conjunto de conhecimentos adquiridos à proporção que lemos diversos tipos de textos e efetuamos correlação entre eles.

Esses conjuntos de ações cognitivas são tratados separadamente para melhor compreensão da sua atuação. Entretanto, são utilizados simultaneamente, não existindo divisão em seu uso, mesmo que não tomemos conhecimento consciente desses atributos. Esses modelos mentais são determinados culturalmente e apreendidos a partir de nossa vivência em sociedade.

Ao estudarem a compreensão textual, os pesquisadores observaram que, quando lemos, procuramos manter o maior número de informações possíveis na memória, objetivando a compreensão do texto. Mesmo assim, não 
buscamos armazenar as palavras exatas, mas tentamos extrair as idéias fundamentais de grupos de palavras e armazená-las, de modo a recuperá-las posteriormente. As formas como as idéias se apresentam são denominadas proposições. As proposições são expressas verbalmente em linguagem natural e, por isso, podem ser avaliadas como verdadeiras ou falsas quando são estudadas. Uma vez que a memória de trabalho preserva as proposições, seus limites não são as palavras, mas as proposições. Além disso, quanto maior for o tempo em que a informação perdure na memória de trabalho, de melhor forma ela será incorporada e mais facilmente será recuperada quando se fizer necessário. Como a memória de trabalho é limitada, é indispensável o apagamento de algumas informações consideradas supérfluas, dando lugar a novas informações.

Na busca de um modelo de processamento de discurso, estudos como o de Kintsch e Van Dijk (1983) incluíam também um modelo de produção por meio da análise e da síntese denominado modelo situacional, ou seja, um modelo que fosse comum a todos os leitores. Os autores observaram que as proposições tematicamente essenciais para a compreensão do texto são conservadas na memória funcional por mais tempo do que aquelas consideradas menos importantes. Kintsch e Van Dijk (1983) denominaram as proposições relevantes "macroproposições", enquanto a estrutura temática do texto é chamada de "macroestrutura". Os pesquisadores investigaram o modelo situacional, solicitando que as pessoas lessem um texto de mil e 300 palavras e o resumissem. Daí detectaram que, mesmo depois de um a três meses, ao serem solicitadas a elaborar novo resumo, lembravam as macroproposições e a macroestrutura geral do texto quase tão bem quanto depois da sua leitura. As proposições que não faziam parte do tema principal do texto foram bem menos detalhadas após um mês e completamente esquecidas após três meses.

Os estudos relacionados com a cognição humana desenvolvidos pelos teóricos das diversas áreas que abordam a questão da leitura e compreensão textual apontam para uma tendência de investigações sobre a importância do conhecimento prévio, as estratégias de transformação da informação em conhecimento e as variáveis textuais, entre outras. Tais abordagens vêm ao encontro das necessidades de diversas áreas do conhecimento, pois, a partir da melhor compreensão da cognição humana, poder-se-á prover melhor as necessidades dos interlocutores dos sistemas de informação.

\section{INTERFACES ENTRE PSICOLOGIA COGNITIVA E CIÊNCIA DA INFORMAÇÃO}

A pesquisa empírica com o enfoque cognitivo na ciência da informação tem evidenciado estudos que convergem mais enfaticamente para a recuperação da informação. Esse foco deve-se à expansão do modelo, denominado por Jacob e Shaw (1998) "quase-paradigma", do ponto de vista cognitivo, defendido primeiramente por De Mey (1982, p. 4), ao asseverar que:

O ponto de vista cognitivo da ciência da informação implica que cada ato de processamento da informação, seja ele perceptivo ou simbólico, é mediado por um sistema de categorias e conceitos os quais, para o mecanismo de processamento da informação, constituem um modelo de mundo.

Seja na recuperação ou no processamento técnico da informação, esse conceito agrega todas as ações realizadas pelos profissionais da informação envolvendo atividades cognitivas.

Ingwersen $(1982,1993,1996)$ tem efetuado pesquisas da cognição humana em sua interação com os sistemas de recuperação da informação. Nesse sentido, o autor (1982, p.167) indica algumas etapas no percurso que leva à recuperação da informação, apresentando os processos cognitivos vivenciados pelos usuários. A necessidade de informação leva o usuário a formular questão(es) ao sistema, que são negociadas entre esse usuário e o bibliotecário. Para responder às questões, o profissional da informação estabelece uma estratégia de busca, seja alfabética ou sistemática, que levará à escolha das ferramentas a serem utilizadas. Além disso, o bibliotecário disponibilizará ao usuário a descrição dos documentos baseada em resumos e títulos, visando à avaliação pelo usuário. Como se pode perceber, a interação entre o usuário e o bibliotecário perpassa pela gestão cognitiva de diferentes modelos de mundo aliados no momento da busca pela informação.

Ao pensarmos um sistema de informação, percebemos que não apenas a recuperação da informação envolve atividades cognitivas. A indexação é um processo intelectual que tem por base a compreensão do texto e a representação do documento, esta intimamente ligada à abordagem do processamento da informação na psicologia cognitiva. Nesse sentido, as pesquisas têm evidenciado três pontos da atividade: compreensão textual, geração de texto (tradução para a linguagem de indexação e/ou resumo) e a representação do conteúdo (criação de linguagens de indexação). 
A abordagem da psicologia cognitiva do processamento da informação vem ao encontro das pesquisas voltadas para o processo de indexação. Para Hjørland (1998), a análise de assunto é uma das atividades fundamentais dos profissionais da biblioteconomia e da ciência da informação. Este autor considera que o momento mais crucial do processo de indexação é a análise de assunto. Tal análise está revestida da subjetividade inerente à política de indexação, que determina prioridades a alguns assuntos.

Outros estudos têm salientado a abordagem cognitiva centrada no processo de indexação, como Beghtol (1986) e Pinto Molina (1994); outros autores apontam para importância do modelo situacional estudado por Kintsch e Van Dijk (1983). Esse modelo indica caminhos viáveis para dinamizar os processos de representação informacional.

O estudo de Kintsch e Van Dijk (1983) tem chamado a atenção dos pesquisadores, por salientar que os leitores proficientes utilizam esquemas psicolingüísticos e operações mentais. Contudo, o discurso permanece inteiro, intacto. Segundo a teoria de Kintsch e Van Dijk (1978), os leitores promovem a desmontagem do texto, lançando mão de regras de "apagamento" (deletion rule) das informações que lhes parecem supérfluas, retendo apenas aquelas importantes. Utilizam também regras de "generalização" (generalisation rules) que permitem reduzir a quantidade de informações semelhantes, proporcionando inferências combinadas e integradas ao texto. Esse exercício possibilita a construção de proposições mais efetivas (construction rules). Tal enfoque compreende a ação dos leitores proficientes de um modo geral. Contudo, essa perspectiva pode tornar-se útil no estudo sobre a leitura para fins de indexação e resumo.

Pesquisa realizada por Fujita (1999a) sobre os procedimentos de leitura documentária estudou a leitura de indexadores, buscando caracterizar o processo de indexação. Ao fazer isso, a autora identificou o uso de estratégias metacognitivas na condução da leitura realizada para fins de representação informacional. Neves (2004) investigou as estratégias de leitura usadas pelo indexador em comparação com outros leitores proficientes. Nessa pesquisa, observou que, apesar de ser um leitor muito habilidoso, o indexador poderia ser instruído durante a sua formação no uso de estratégias metacognitivas de leitura que viabilizassem maior eficiência ao seu desempenho durante o processo de indexação.

Os estudos referidos neste trabalho são apenas alguns exemplos de pesquisas que demonstram a preocupação dos teóricos da ciência da informação em compreender de forma mais ampla a cognição humana. Preocupação essa salientada por Morin (1999, p. 40): “... o operador do conhecimento deve tornar-se ao mesmo tempo objeto do conhecimento".

\section{CONSIDERAÇÕES FINAIS}

Nas diversas áreas do conhecimento, está ocorrendo, desde meados do século XX, um movimento em direção a teorias relativamente recentes que focalizam a emergência de pesquisas sobre o desempenho mental dos indivíduos. Pretende-se resposta para a inquietante questão: como a nossa mente funciona? E não se busca saber apenas o funcionamento fisiológico do cérebro, mas os meandros da origem do comportamento e atitudes dos seres humanos.

No que diz respeito à ciência da informação, do nosso ponto de vista, a adoção da abordagem processamento da informação vem ao encontro de estudos que envolvem a cognição na recuperação e no processamento técnico da informação. Os estudos focalizados no presente artigo dão uma pequena demonstração das possibilidades que se abrem diante das inúmeras possibilidades das descobertas de estudos transdisciplinares.

O desenvolvimento de habilidades do ponto de vista da qualificação profissional do bibliotecário demanda maior ênfase, para que os profissionais da indexação possam ter contato com esse universo de práticas cognitivas. Assim, a inserção desse conhecimento em componentes curriculares na formação acadêmica do profissional da informação possibilitaria uma orientação pontual para a relevância dos processos cognitivos no desenvolvimento de experiências monitoradas de leitura para o futuro indexador de textos, como também na interação com o usuário.

Artigo submetido em 05/08/2005 e aceito em 25/05/2005. 


\section{Dulce Amélia Neves}

\section{REFERÊNCIAS}

BEGHTOL, Clare. Bibliographic classification theory and text linguistics: aboutness analysis, intertextuality and the cognitive act of classifying documents. Journal of Documentation, v. 42, n. 2, p. 84113, June 1986.

CAPURRO, Rafael. Foundations of information science: review and perspectives. In: INTERNATIONAL CONFERENCE ON CONCEPTIONS OF LIBRARY AND INFORMATION SCIENCE, 1991, Tampere. Electronic Proceedings... Tampere: University of Tampere, 1991. Disponível em: < http://www.capurro.de/tampere91.htm $>$. Acesso em: 2006.

CINTRA, Anna M. M. et al. Para entender as linguagens documentárias. São Paulo: Polis: APB, 1994.

DAHLBERG, Ingetraut. Teoria do conceito. Ciência da Informação, Brasília, v. 7, n. 2, p. 101-107, 1978.

DE MEY, M. The cognitive paradigm: an integrated understanding of science development. Chicago: University of Chicago, 1982.

ECO, Umberto. Interpretação e superinterpretação. São Paulo: Martins Fontes, 1997. p.79-104.

EYSENCK, Michael W.; KEANE, Mark T. Psicologia cognitiva: manual introdutório. Porto Alegre: Artes Médicas, 1994.

FLAVELL, J. H. Metacognition and cognitive monitoring: a new area of cognitive-developmental inquiry. American Psychologist, v. 34, p. 906-911, 1979.

; MILlER, P. H.; MILLER, S. A. Desenvolvimento cognitivo. 3. ed. Porto Alegre: Artmed, 1999.

FUJITA, Mariângela S; NARDI, Maria Izabel A; SANTOS, Silvana. A leitura em análise documentária. Transinformação, v. 10, n. 3, set./ dez. 1998. Disponível em: <http://www.puccamp.br/ biblio/ transinformacao/old/vol10n3/pag13.html >. Acesso em: 2006.

. A leitura do indexador: estudo de observação. Perspectiva em Ciência da Informação, v. 4, n. 1, p. 101-116, jan./jun. 1999a.

- Análise e síntese documentárias para a compreensão de leitura de textos didáticos: uma proposta de aplicação do sistema de indexação PRECIS. Informare, v. 5, n. 1, p. 77-94, jul./dez. 1999b.

HJ $\varnothing$ RLAND, B. Theory and metatheory of information science: a new interpretation Journal of Documentation, v. 54, n. 5, p. 606-621, 1998. Disponível em http://www.db.dk/bh/publikationer/filer/theory\% 20and\%20metatheory.pdf Acesso em: 22/09/2005.

HUNT, E. Intelligence as an information processing concept. British Journal of Psychology, v. 71, p. 449-474, 1980.

INGWERSEN, Peter. Search procedures in the library; analyzed from the cognitive point of view. Journal of Documentation, v. 38, n.3, p. 165 191, Sept. 1982.

1992.

. Information retrieval interaction. London: Taylor Graham,

Cognitive perspectives of information retrieval interaction: elements of a cognitive IR theory. Journal of Documentation, v. 52, n. 1, p. 3-50, 1996. Disponível em: < http://www.db.dk/pi/iri/ \#chapters $>$. Acesso em: 2006.

JACOB, Elin K.; SHAW, D. Sociognitive perspectives on representation. Annual Review of Information Science and Technology, v. 33, p. 131-185, 1998.
JOHNSON-LAIRD, P. Mental models. Cambridge, MA: Harvard University Press, 1983.

KINTSCH, Walter, VAN DIJK, Teun A. Strategies of discourse comprehension. New York: Academic Press, 1983.

Toward a model of text comprehension and production. Psychological Review, v. 85, n. 5, p. 363-394, Sep. 1978.

KLEIMAN, Ângela. Texto e leitor: aspectos cognitivos da leitura. Campinas: Pontes, 1995.

KOCH, Igedore G. Villaça; TRAVAGLIA, Luiz Carlos. A coerência textual. 11. ed. São Paulo: Contexto, 2001.

LIMA, Gercina A. Borém. Interfaces entre ciência da informação e ciência cognitiva. Ciência da Informação, v. 32, n. 1, p.77-87 jan./abr. 2003. Disponível em: < http://www.ibict.br/cienciadainformacao/ viewarticle.php?id=166\&layout=abstract $>$. Acesso em: 2006.

NEVES, Dulce Amélia de B. Aspectos metacognitivos na leitura do indexador. 2004. Tese (Doutorado em Ciência da Informação)- UFMG, Belo Horizonte, 2004.

; CRUZ, Emília B. Transacionando com os campos do saber. In: ENCONTRO NACIONAL DE PESQUISA EM CIÊNCIA DA INFORMAÇÃO, 4., 2000, Brasília. Anais... Brasília: UnB, 2000.

; MOURA, Maria A. Ciência da informação, semiótica e cognição: interseções. Athos $\mathscr{E}$ Ethos, v. 2, p. 175-198, 2002.

NEWELL, A.; SIMON, H. A. Human problem solving. Englewood Cliffs, NJ: Prentice-Hall, 1972.

PIAGET, Jean. A epistemologia genética: sabedoria e ilusões da filosofia, problemas de psicologia genética. 2. ed. São Paulo: Abril Cultural, 1983. (Os pensadores).

- Aprendizagem e conhecimento. 9. ed. Rio de Janeiro: Martins Fontes, 1998.

Psicologia e pedagogia. São Paulo: Forense Universitari, 2003.

PINTO MOLINA, Maria. Interdisciplinary approaches to the concept and practice of written text documentary content text. Journal of Documentation, v. 50, n. 2, p. 111-133, June 1994.

SARACEVIC, T. Ciência da Informação: origem, evolução e relações. Perspectivas em Ciência da Informação, Belo Horizonte, v. 1, n. 1, p. 41 62, jan./jun. 1996.

STERNBERG, R.J. A component process in analogical reasoning. Psychological Review, n. 84, p. 353-378, 1977.

The nature of mental abilities. American Psychologist, n. 34, p. 214-230, 1979.

p. $1-48,1983$.

Components of human intelligence. Cognition, n. 15, . Psicologia cognitiva. Porto Alegre: Artmed, 2000.

VAN DIJK, Teun A. Cognição, discurso e interação. 2. ed. São Paulo: Contexto, 1999.

VYGOTSKI, Lev S.; LURIA, Alexander R.; LEONTIEV, Alexis N. Linguagem, desenvolvimento e aprendizagem. São Paulo: Ícone, 1998. 